EPJ Web of Conferences 40, 17002 (2013)

DOI: $10.1051 /$ epjconf/20134017002

(C) Owned by the authors, published by EDP Sciences, 2013

\title{
Structure and magnetic properties of FeNi/Ti sputtered multilayers
}

\author{
N. Villar Alzola ${ }^{1}$, A.V. Svalov ${ }^{1,2}$, N.S. Mayura ${ }^{2}$, N.A. Kulesh ${ }^{2}$, A. Larrañaga ${ }^{3}$, and G.V. Kurlyandskaya ${ }^{1,2}$ \\ ${ }^{1}$ Departamento de Electricidad y Electrónica, Universidad del País Vasco, 48080 Bilbao, Spain \\ ${ }^{2}$ Department of Magnetism and Magnetic Nanomaterials, Ural Federal University, 620002 Ekaterinburg, Russia \\ ${ }^{3}$ SGIker, Servicios Generales de Investigación, Universidad del País Vasco, 48080 Bilbao, Spain
}

\begin{abstract}
The microstructure, anisotropic magnetoresistance, magnetic properties and magnetic domain structure of sputtered FeNi films and $[\mathrm{Ti} / \mathrm{FeNi}]_{\mathrm{n}}(\mathrm{n}=2-16)$ multilayers were comparatively analyzed. It was found that although the grain size increases with an increase of the FeNi thickness both in the case of FeNi films and $[\mathrm{Ti} / \mathrm{FeNi}]_{\mathrm{n}}$ multilayers, it did not exceed $25 \mathrm{~nm}$. The values of anisotropic magnetoresistance for FeNi films and $[\mathrm{Ti} / \mathrm{FeNi}]_{\mathrm{n}}$ multilayers were close to each other showing a weak dependence on the total thickness of the multilayered structure. Coercivity for multilayers was found to be smaller than the coercivity of single layer FeNi films. Despite the absence of a direct exchange interaction between FeNi neighboring layers in the $[\mathrm{Ti} / \mathrm{FeNi}]_{\mathrm{n}}$ structures, their domain structures were found to be quite different from magnetic domains in single layer films due to stray field compensation in the multilayers. Obtained results are useful for the development of sensitive elements for small magnetic field detectors and planar inductors.
\end{abstract}

\section{Introduction}

Thin permalloy films are versatile materials suitable for different technological applications such as giant magnetoimpedance based magnetic field detectors, planar inductors, etc. [1-2]. Their main technologically requested parameters are high magnetic permeability and low coercivity. It is known that laminating a thick magnetic film with a nonmagnetic spacer may further improve the magnetic softness of the thin film based structure. In multilayeres, strong magnetostatic interactions between the domain walls situated in different magnetic layers are possible. In this case, domain boundaries may form paired domain wall structures with a partially closed magnetic flux and reduced energy, causing, as a consequence, a reduction of the coercivity of the multilayers [3]. Non-magnetic spacer introduction changes the thickness of magnetic layers and as a consequence can affect the crystalline structure of the layers because such parameters as the average grain size, depend on the layer thickness [4]. The average grain size is directly connected with the magnetic softness of thin films. In addition, a non-magnetic spacer plays the role of a seed layer for the next deposited magnetic layer, affecting the magnetic properties through a change in film structure. The magnetic properties of sputtered thin films can be improved through deposition onto an appropriate seed layer [5]. Thus, there are different important mechanisms playing a decisive role with respect to magnetic properties of magnetic multilayers with non-magnetic spacers.

In this work the microstructure, anisotropic magnetoresistance (AMR), magnetic properties and magnetic domains were comparatively analyzed for $\mathrm{Ti} / \mathrm{FeNi}$ films and $[\mathrm{Ti} / \mathrm{FeNi}]_{\mathrm{n}}$ multilayers.

\section{Experimental}

Ti/FeNi films (single-layered FeNi films deposited onto Ti seed layer) and $[\mathrm{Ti} / \mathrm{FeNi}]_{\mathrm{n}}(\mathrm{n}=2-16)$ multilayers were prepared by alternative magnetron sputtering deposition of FeNi layers and Ti seed layers or spacers onto glass substrates. The base pressure in the vacuum chamber was $3 \times 10^{-7}$ mbar and an argon gas flow with a pressure of $3.8 \times 10^{-3}$ mbar was used for the sputtering process. The deposition rates were about $0.4 \mathrm{~nm} / \mathrm{s}$ for $\mathrm{FeNi}$ and about $0.07 \mathrm{~nm} / \mathrm{s}$ for $\mathrm{Ti}$ layers. The thicknesses of the $\mathrm{FeNi}$ layers $\left(L_{\mathrm{FeNi}}\right)$ were varied in the interval of 12 to $200 \mathrm{~nm}$ and the thickness of $\mathrm{Ti}$ non-magnetic layers was kept constant $(6 \mathrm{~nm})$. A magnetic field of 250 Oe was applied parallel to the film plane during deposition in order to induce a uniaxial magnetic anisotropy.

The microstructure of the samples was studied by Xray diffraction (XRD) using $\mathrm{Cu} K_{\alpha}$ radiation. The magnetoresistance was measured following the conventional four-point probe technique. The AMR ratio was defined as follows: $\Delta R / R=100 \times\left[\left(R_{||}-R_{\perp}\right) / R_{||}\right]$, where $R_{\|}$and $R_{\perp}$ are the electrical resistance parallel and perpendicular to the magnetization vector, respectively. The current was directed in the film plane along the easy 


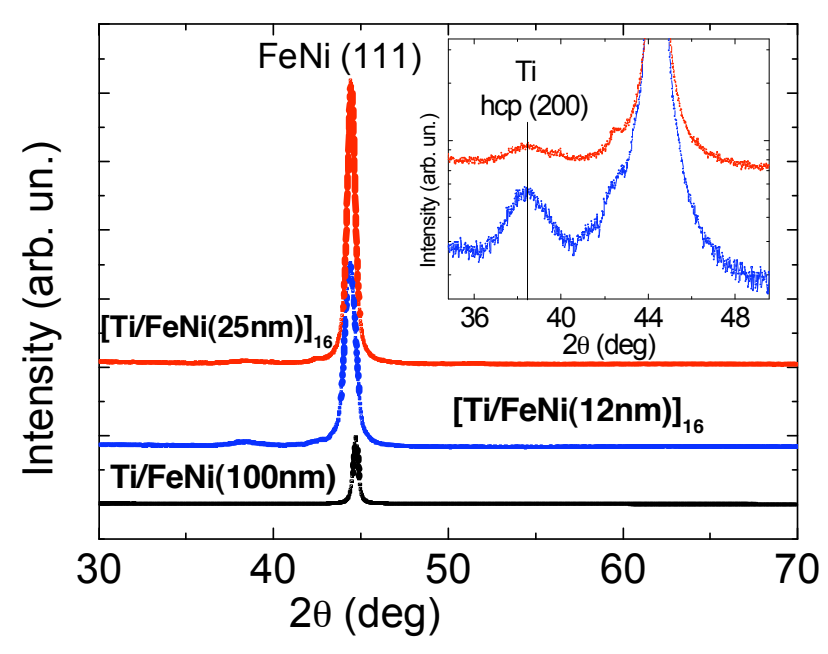

Fig. 1. X-ray diffraction spectra of selected FeNi-based structures. Inset shows the maxima at lower scale.

axis of the sample. The $R_{\|}$value was measured at the magnetic remanence state. Magnetic hysteresis curves with the applied field in plane were measured by a vibrating sample magnetometer. The magnetic domain structure was studied by the optical microscopy in remanence state using the standard Bitter technique [6].

\section{Results and discussion}

As an example, figure 1 shows the $\theta-2 \theta$ XRD spectra of selected samples. The main common feature observed in all samples is the visualization of (111) - the only peak of the fcc FeNi indicating the high texturing level of the crystallographic texture of permalloy layers. When the thickness of the FeNi layers of the multilayered structure goes down (as the number of layers and Ti spacers goes up) one can appreciate Ti hcp (200) peak of the XRD spectra of $[\mathrm{Ti} / \mathrm{FeNi}]_{\mathrm{n}}$ multilayers. Thus, Ti layers in the present study have a hcp structure.

For all samples the grain size was calculated using Scherrer's equation. Both for Ti/FeNi and $[\mathrm{Ti} / \mathrm{FeNi}]_{\mathrm{n}}$ samples the grain size of the FeNi layers is equal to layer thickness at $L_{\mathrm{FeNi}}$ of 12 and $25 \mathrm{~nm}$. For the other $L_{\mathrm{FeNi}}$ the average size is constant being approximately $25 \mathrm{~nm}$. These data are also confirmed by the results of the measurements of the dependence of the AMR ratio on the thickness of FeNi layers (figure 2).

Initial increase in $\Delta \mathrm{R} / \mathrm{R}$ with FeNi thickness increase is due to the augment of the average grain size - the resistivity of FeNi layers decreases with an increase of the grain size due to a reduction in the grain boundary scattering of conduction electrons [5]. As the thickness further increases the $\Delta R / R$ stays constant as a consequence of the constant average size of the grains for $L_{\mathrm{FeNi}}>25 \mathrm{~nm}$, which approximately coincides with mean free path of electrons in FeNi thin films $(\sim 30 \mathrm{~nm})$ [7]. The higher values of the AMR for multilayered structures with $L_{\mathrm{FeNi}}<50 \mathrm{~nm}$ can be understood if one takes into account their higher total thickness and therefore lower resistance value. This additional possibility to control resistance of thin film or multilayers can be useful for the

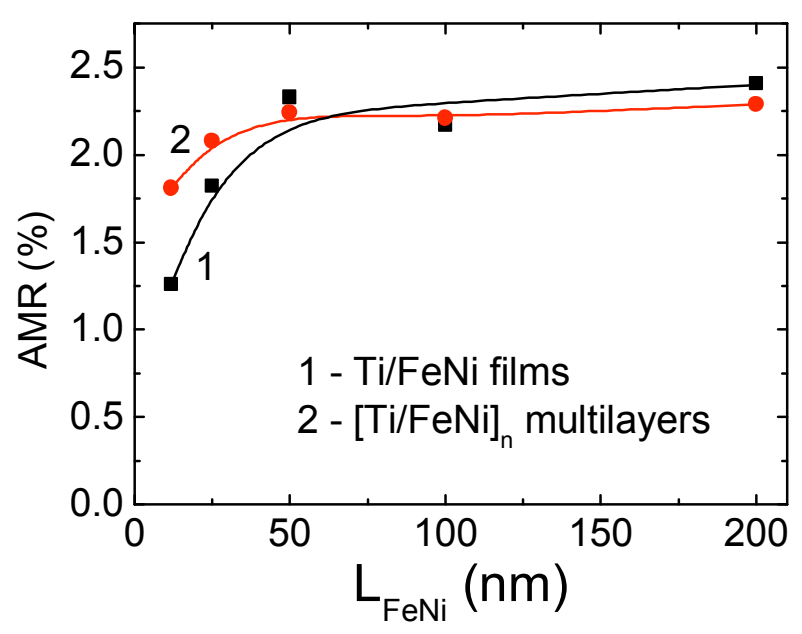

Fig. 2. The dependence of AMR values on FeNi thickness for $\mathrm{Ti} / \mathrm{FeNi}$ films and $[\mathrm{Ti} / \mathrm{FeNi}]_{\mathrm{n}}$ multilayers.

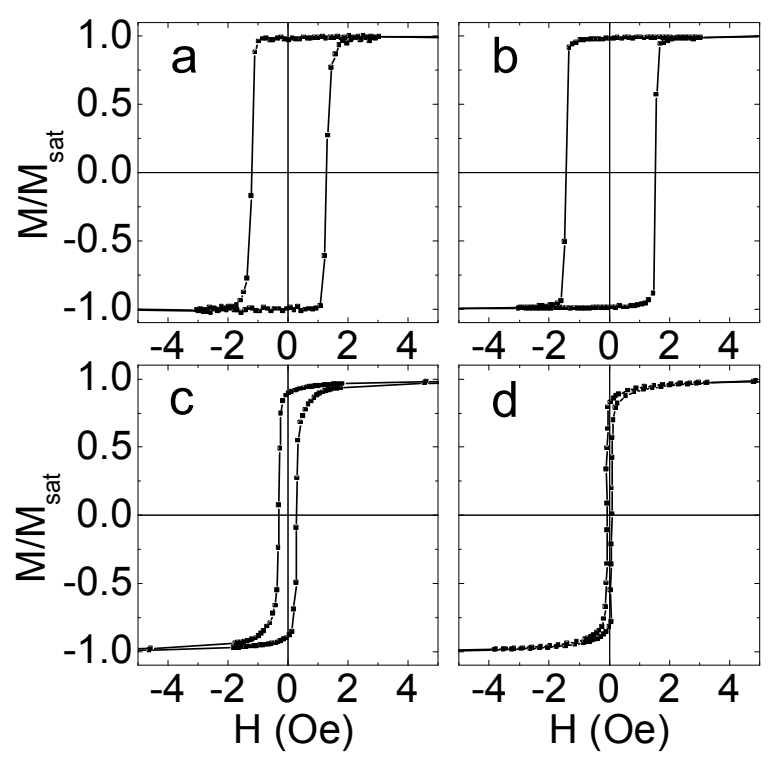

Fig. 3. Magnetization curves along the easy axis for selected samples: Ti/FeNi(12nm) - (a), Ti/FeNi(100nm) - (b), $[\mathrm{Ti} / \mathrm{FeNi}(12 \mathrm{~nm})]_{16}-(\mathrm{c})$ and $[\mathrm{Ti} / \mathrm{FeNi}(100 \mathrm{~nm})]_{4}-(\mathrm{d})$.

design of particular alternating current carrying circuits with a thin film based sensitive element where an impedance match is very essential [2].

It is known that grain size is an important parameter for the thin film magnetization process. Figure 3 shows magnetization curves of the samples measured parallel to the easy magnetization axis by VSM for selected Ti/FeNi films and $[\mathrm{Ti} / \mathrm{FeNi}]_{\mathrm{n}}$ multilayers. In both cases the magnetization curves show high remanence and squareness. The dependence of coercivity $\left(H_{c}\right)$ versus FeNi thickness is shown in figure 4 . It is seen that one layered films and multylayers have different $H_{\mathrm{c}}\left(L_{\mathrm{FeNi}}\right)$ dependencies, both of them differing from the dependence of the average grain size on the $\mathrm{FeNi}$ thickness described above in this section.

For small $L_{\mathrm{FeNi}}$ values $H_{\mathrm{c}}$ decreases but it increases at $L_{\mathrm{FeNi}}>180 \mathrm{~nm}$ in both types of samples. The first feature is a consequence of a decrease of the relative influence of the FeNi layer surface in the course of the 


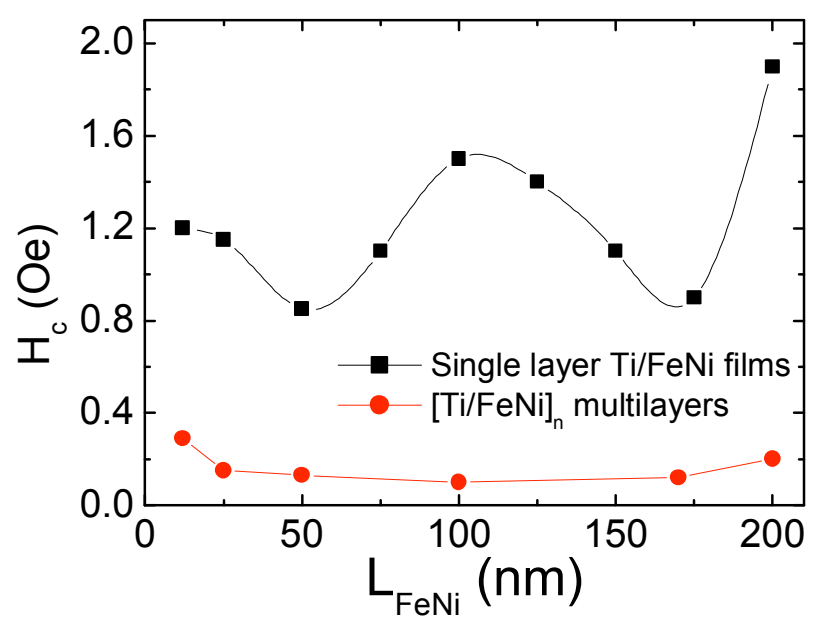

$L_{\mathrm{FeNi}}$ Fig. 4. The dependence of coercivity versus $\mathrm{FeNi}$ thickness for $\mathrm{Ti} / \mathrm{FeNi}$ films and $[\mathrm{Ti} / \mathrm{FeNi}]_{\mathrm{n}}$ multilayers.

increase. The second feature is due to the beginning of the transition of the FeNi layers into a "transcritical" state always resulting in the coercivity increase [8-9]. The appearance of the $H_{c}$ peak at $L_{\mathrm{FeNi}} \sim 100 \mathrm{~nm}$ for Ti/FeNi films seems to be connected with a transition of the structure of the domain walls caused by an increase of the FeNi film thickness.

Figure 5 shows the evolution of the magnetic domain structure as a function of the FeNi film thickness which well coincide with specific features of the $H_{c}$ behaviour as a function of the permalloy layer thickness. Very thin (about $12 \mathrm{~nm}$ ) films with in-plane magnetization as usual contain pure Néel walls with segments of walls having different rotation senses. Magnetizations inside the magnetic domains, for all thin films under consideration, lie in-plane of the film. Thin films of about $50 \mathrm{~nm}$ thick are Néel type walls with cross-tie specific features [6, 10] which are clearly seen in figure 5 (b). In the case of 100 $\mathrm{nm}$ thick films (figure 5 (c)) two types of domain walls co-exist being neighbour parts of the same domain wall. Most probably they are $180^{\circ}$ Néel walls with cross ties and stray field free asymmetric mobile vortex Bloch walls [7].

If $L_{\mathrm{FeNi}}$ further increases (above $100 \mathrm{~nm}$, Fig. 5(d)) the only type of walls observed are Bloch walls. Thereby, the grain size probably contributes to $H_{c}$ value, but precisely the domain walls transformation plays a decisive role changing their characteristics like a mobility and longshort interaction range in the $\mathrm{FeNi}$ films finally determining the most important contribution in $H_{c}$. The most realistic scenario for co-existence of two types of domain walls seems to be at $L_{\mathrm{FeNi}} \sim 100 \mathrm{~nm}$. At the same time this leads to an increased hysteresis and opposition to the domain walls' displacements.

The situation changes completely in the case of magnetic multilayered structures. As an example, figure 5(e) shows the magnetic domain structure for [Ti(6 $\mathrm{nm}) / \mathrm{FeNi}(100 \mathrm{~nm})]_{4}$ multilayers. Instead of cross tie walls with their long-range interactions (figure 5(b)) one can see a very uniform magnetic domain structure with non-charged walls and wide domains. Additional confirmation of exact in-plane magnetization orientation

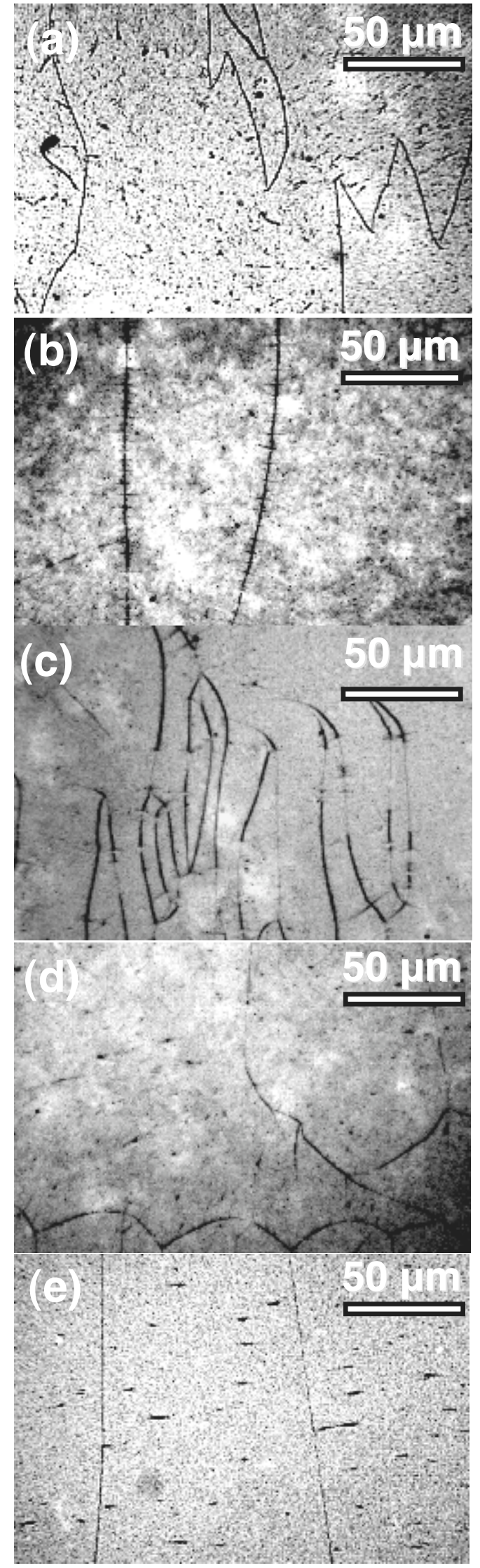

Fig. 5. Magnetic domain structure of $\mathrm{Ti} / \mathrm{FeNi}$ films (a-d) and $[\mathrm{Ti} / \mathrm{FeNi}(100 \mathrm{~nm})]_{4}$ multilayer (e) in remanence revealed by Bitter technique. In-plane direction of the easy magnetization axis is vertical. The thickness of FeNi layer is $12 \mathrm{~nm}$ - (a), 50 $\mathrm{nm}-(\mathrm{b}), 100 \mathrm{~nm}-$ (c) and $175 \mathrm{~nm}-$ (d). 
comes from the rather specific contrast inside each domain, namely, typical short elongated accumulations of ferrofluid observed in the direction perpendicular to the magnetization orientation [11]. Such a dramatic change was caused by positive (ferromagnetic) coupling between the FeNi layers together with the magnetostatic interactions between the layers caused by long-range interactions of the cross-tie containing walls. It is logical to suppose that the domain walls situated in different magnetic layers form magnetic structures with a closed or partially closed magnetic flux. Such a structure formation can be associated with a decrease of the energy density corresponding to the domain wall and as a consequence results in a decrease of the coercivity $[3,12]$.

Of course, more precise micromodelization is necessary in order to understand the thickness dependence of the structure of the paired domain walls. However, in any case the formation of the magnetic multilayered structures with a partially closed magnetic flux results in the change of domain wall structure and diminishes the coercivity of $[\mathrm{Ti} / \mathrm{FeNi}]_{\mathrm{n}}$ multilayers: $H_{c}$ of $[\mathrm{Ti} / \mathrm{FeNi}]_{\mathrm{n}}$ multilayers is less than coercivity of $\mathrm{Ti} / \mathrm{FeNi}$ films for all $L_{\mathrm{FeNi}}$ values.

Coming back to the results of the structural studies and taking into account some previous studies of the dynamic properties of $[\mathrm{Ti} / \mathrm{FeNi}]_{\mathrm{n}}$ multilayers (ferromagnetic resonance, FMR, [13]) we can roughly estimate the opposed tendencies caused by nanostructuring of thick permalloy films by the Ti spacer. It was shown that values of the resonance fields of the $[\mathrm{Ti} / \mathrm{FeNi}]_{\mathrm{n}}$ multilayers were quite close to the corresponding values for the FeNi films prepared in the same conditions [13]. This indicated that obtained $[\mathrm{Ti} / \mathrm{FeNi}]_{\mathrm{n}}$ multilayers were homogeneous ferromagnets with no significant contribution of the perpendicular magnetic anisotropy component. At the same time the observed slight increase of the resonance line widths for $[\mathrm{Ti} / \mathrm{FeNi}]_{\mathrm{n}}$ multilayers with an increase of the number of non-magnetic spacers confirmed the existence of the process of gradual accumulation of magnetic inhomogeneities in the system. In a most simple way one can connect the increase of the degree of inhomogeneity with an increase of the number of interfaces and possible increase of the volume of mixed interfaces. Indeed, very careful XRD analysis of Ti/FeNi multilayers [14] had shown the possibility of detection of (FeNi)Ti cubic phase with smaller value of cell parameter. Even so the possibility of formation of magnetic structures with a partially closed magnetic flux is dominating and decisive for obtaining low coercivity.

\section{Conclusion}

$\mathrm{Ti} / \mathrm{FeNi}$ films and $[\mathrm{Ti} / \mathrm{FeNi}]_{\mathrm{n}}$ multilayers were prepared by magnetron sputtering. A comparative analysis of the structure, anisotropic magnetoresistance, magnetic properties, and magnetic domains was conducted. In both cases of single layered films and multilayered nanostructures the grain size of the FeNi layers is equal to layer thickness at $L_{\mathrm{FeNi}}$ of $12 \mathrm{~nm}$ to $25 \mathrm{~nm}$. For other $L_{\mathrm{FeNi}}$ thicknesses the average size is constant being approximately $25 \mathrm{~nm}$. The dependence of the AMR effect value on the $L_{\mathrm{FeNi}}$ thickness correlates well with the dependence of the average grain size for both types of samples.

Coercivity of prepared multilayers was found to be smaller than the coercivity of FeNi single layer films for all $L_{\mathrm{FeNi}}$. We suppose that this tendency can be explained by the formation of paired domain walls situated in the adjacent FeNi layers with a partially closed magnetic flux. The results obtained in the present study should help optimize the parameters necessary for the on purpose use of the FeNi-based magnetic nanostructures in high frequency technological applications.

\section{Acknowledgments}

This work was supported by MAT2011-27573-C04 project of the Spanish Government, Ural Federal University (Grant 215), and the Ministry of Education and Science of Russian Federation (Contract 16.552.11.7020). Selected measurements were done in SGIKER Services, UPV-EHU. We thank Iñaki Orue and Anna Svalova for special support.

\section{References}

1. D.S. Gardner, G. Schrom, F. Paillet, B. Jamieson, T. Karnik, and S. Borkar, IEEE Trans. Magn., 45, 4760 (2009)

2. G.V. Kurlyandskaya, D. de Cos, S.O. Volchkov, Rus. J. Nondestr. Test., 45, 377 (2009)

3. E. Feldtkeller, J. Appl. Phys. 39, 1181 (1968)

4. D.G. Neerinck, A.E.M. De Veirman, M.H.J. Slangen, Th.G.S.M. Rijks, J.C.S. Kools, Thin Solid Films, 280, 136 (1996)

5. W.Y. Lee, M.F. Toney, D. Mauri, IEEE Trans. Magn., 36, 381 (2000)

6. A. Hubert and R. Schäfer, Magnetic Domains (Springer, Berlin, 1998)

7. S. Tumanski, Thin Film Magnetoresistive Sensors (IOP Publishing, Bristol, 2001)

8. M. Coïsson, F. Vinai, P. Tiberto, and F. Celegato, J. Magn. Magn. Mater., 321, 809 (2009)

9. A.V. Svalov, I.R. Aseguinolaza, A. Garcia-Arribas, I. Orue, J.M. Barandiaran, J. Alonso, M.L. FdezGubieda, G.V. Kurlyandskaya, IEEE Trans. Magn., 46, 333 (2010)

10. E. Feldtkeller, J. Phys. Colloques 32, C1-452 (1971)

11. R. Carey, E.D. Isaac, Magnetic Domains and Techniques for Their Observation (Academic Press, New York, 1966)

12. V.O. Vas'kovskii, P.A. Savin, V.N. Lepalovskii, A.A. Ryazantsev, Phys. Solid State 39, 1958 (1997)

13. G.V. Kurlyandskaya, S.M. Bhagat, A.V. Svalov, E. Fernandez, A. Garcia-Arribas1, J.M. Barandiaran Sol. State Phenom., 168-169, 257 (2011)

14. N. Villar Alzola, G.V. Kurlyandskaya, A. Larrañaga, A.V. Svalov, IEEE Trans. Magn., 48, 1605 (2012) 